\title{
A Reliable and Robust QoS Routing Protocol for Mobile Wireless Sensor Networks
}

\author{
Suresh Chimkode \\ Asst. Prof \\ Dept. of CSE. \\ GND College of Engg. \\ Bidar,India
}

\author{
Ramesh S. Jadhav \\ Lecturer \\ Dept. of CSE. \\ Appa IET, Gulbarga. India
}

\author{
Basavraj S. Mathapati \\ Dept. of CSE \\ Dept. of CSE. \\ Appa IET, Gulbarga, India
}

\begin{abstract}
The problem of guaranteeing Quality-of-Service (QoS) routing in wireless sensor networks (WSNs) with node mobility is a difficult problem, even without possible interference from external radio sources. Difficulties arise because node mobility can cause frequent network topology changes, communication channels can have high error rates, the jitter rate is high and several different applications can be sharing the use of the communication medium. In this paper we propose a reliable and robust QoS routing protocol for WSNs based on a Combined Weight $(\mathrm{CW})$ Value. The $\mathrm{CW}$ is based on the QoS parameters link quality, residual energy and available bandwidth. In addition to this, we propose an adaptive rate control mechanism in order to avoid congestion losses. By simulation results, we show that our proposed protocol is reliable and robust when compared to existing routing protocol.
\end{abstract}

\section{General Terms}

Combined Weight, Adaptive Rate.

\section{Keywords}

Qos, WSN, Route Request, QBRP.

\section{INTRODUCTION}

A wireless sensor network is a collection of nodes organized into a cooperative network [1]. A wireless sensor network (WSN) is a network that is made of hundreds or thousands of sensor nodes which are densely deployed in an unattended environment with the capabilities of sensing, wireless communications and computations [2]. The most important three characteristics of WSN are (i) Sensor nodes are prone to maximum failures, (ii) Sensor nodes make use of the broadcast communication pattern and have severe bandwidth restraint, (iii) Sensor nodes have limited amount of resources [3].

There are some constraints in wireless sensor networks such as memory, low-power consumption, fault-tolerance, lowlatency and coverage. Sensor networks are applied in a wide range of areas, such as military applications, public safety, medical, surveillances, environmental monitoring, commercial applications, habitat and tracking.

QoS is a set of service requirements to be met when transporting a packet stream from the source to its destination [4]. Quality of service (QoS) is defined as "the capability to provide resource assurance and service differentiation in a Network" [5]. Quality of Service (QoS) aims at providing better networking services over current technologies such as ATM, Ethernet and others. The main three parameters for
QoS are latency (delay), jitter and loss. Other QoS parameters include reliability, responsiveness, mobility, power efficiency network availability and bandwidth [6].QoS in wireless sensor networks can be characterized by reliability, timeliness, robustness, availability, and security between all others [7].

QoS protocols in sensor networks have several applications including real time target tracking in battle environments, emergent event triggering in monitoring applications etc [8].

Quality of Service (QoS) requirements in the routing protocols for mixed data reporting applications. Due to the dynamic nature of the network, the existing QoS protocols for wired networks cannot be applied directly to WSNs. Congestion control mechanisms are essential in WSNs [9].

QoS routing requires not only to find a route from a source to a destination, but a route that satisfies the end-to-end QoS requirement, often given in terms of bandwidth, delay or loss probability [10]. Transmission of video and imaging data requires both energy and QoS aware routing in order to ensure efficient usage of the sensors and effective access to the gathered measurements [11]. The advantage of QoS routing protocol becomes apparent when traffic gets heavy. A major criticism of such QoS routing protocol is that it is designed without considering the situation when multiple QoS routes are being setup simultaneously [12].

Source routing has severe disadvantages in Wireless sensor networks with high dynamics, because of the increased communication overhead to exchange information about net state, even when no routes need to be discovered. In wireless networks, there are additional considerations to be taken into account. Node mobility and the wireless nature of communication - prone to noise and dependent on various environmental conditions - affect the connectivity of the network, causing its topology to change, often rather rapidly. This is aggravated by further constraints on energy reserves and available bandwidth and signal degradation by noise and limited transceiver resources. Therefore, instead of a traditional layered network control approach, a joint optimization scheme affecting both the link and the routing layer is necessary. First, we propose a routing algorithm with a QoS Monitoring Agent. In our algorithm, a combined weight $(\mathrm{CW})$ value is assigned to each links which is based on the network parameters link quality, available bandwidth and residual energy. The link quality can be estimated from the node mobility and RSSI. In addition we follow an adaptive rate control mechanism in order to forward the data through an efficient route. 


\section{RELATED WORK}

Mirela Fonoage et al [9] have proposed a QoS based routing protocol with a congestion control mechanism for mixed data reporting WSNs. The congestion control mechanism is targeting the event packets specifically, since the reporting interval is smaller compared to the periodic reporting. Allowing a large number of similar packets to flow in the network may trigger its congestion and a reduction in the network lifetime. The protocol uses a barrier-based congestion control mechanism that allows efficient data aggregation and avoids energy depletion due to heavy reporting.

Sanjay Kumar et al [13] have proposed a QoS based solaraware clustered routing protocol (QSCR) which is an extension to sLEACH for routing. A QoS based Solar Aware Clustered Routing protocol designed for solar aware wireless sensor networks to provide quality of service by performing centralized clustering algorithm. The base station selects the cluster head on the basis of the lifetime of a node and its solar status.

Deepali Virmani et al [14] have proposed a new scheme the stable routing by using virtual nodes for self stabilization with power factor (SRVNP) which would allow sensor nodes to maintain routes to destinations with more stable route selection. This new scheme has been proposed for achieving QoS in terms of packet delivery, multiple connections, better power management and stable routes in case of failure. It offers quick adaptation to distributed processing, dynamic linking, low processing overhead and loop freedom at all times.

Rui Dai et al [15] have proposed a correlation-aware QoS routing algorithm for wireless video sensor networks. Firstly, a correlation-aware differential coding scheme is designed to reduce the amount of traffic generated by correlated video sensors. Then, a correlation-aware load balancing scheme is proposed to prevent network congestion by splitting the correlated flows that cannot be reduced to different paths. Finally, these correlation aware schemes are integrated into an optimization QoS routing framework with an objective to minimize energy consumption subject to QoS constraints.

Manuel Diaz et al [16] have presented a novel directed acyclic graph based QoS-aware non-deterministic routing algorithm. The proposed algorithm uses a non deterministic routing scheme that is less prone to attacks and that balances the energy consumption across the network. It also allows simultaneous use of reliability, deadline, priority and energy efficiency configurations in the communication between the nodes and the sink. The routing algorithm uses a model that improves the reliability between nodes and allows the programmer to specify the desired reliability level quantitatively.

Tenali. Nagamani et al [17] have proposed two algorithms: Reliable-IRL algorithm and Data privacy mechanism. The proposed requires less energy, power and computational power, with more reliability. And provides protection against various attacks likes hop-by-hop trace back, eavesdropping and disclosure attacks. The Reliable-IRL algorithm provides Identity, route and location privacy with direction and trust notations. And data privacy algorithms provides security to data with encryption and adding random number to the pay load field of packet we are providing full network level privacy.

\section{DESIGN OF RELIABLE AND ROBUST QOS ROUTING PROTOCOL 3.1 Overview of Reliable \& Robust QoS Routing Protocol}

We have designed a new QoS based routing protocol for Wireless Sensor Networks. To design a new routing protocol we developed a new routing algorithm, a combined weight (CW) value is assigned to each links which is based on the network parameters link quality, available bandwidth and residual energy. The link quality can be estimated from the node mobility and RSSI. All nodes hold the minimum combined weight value (MCW) together with each routing entry in the routing table. If $\mathrm{CW}$ value is higher than the $\mathrm{MCW}$, only then all the following copies of the Route Request are forwarded. If the value is lower, then the current $\mathrm{MCW}$ is replaced by the lowest value. This guarantees that the Route Request with the lowest congestion and maximum channel utilization is forwarded and employed for route creation.

The monitoring agent sends a setup message along with the traffic flows, to obtain the QoS information required to estimate the $\mathrm{CW}$ value. In case of QoS changes or route breakages, a warning is sent to the source, which contains the estimated amount of resources to be reserved or the route failure information. The sender adaptively adjusts the reservations or data rate when there is a QoS change or selects another efficient route when there is a route or link failure according to this warning information.

\subsection{Estimation of Combined Weight $(\mathrm{CW})$ Value}

\subsubsection{Computation of Available Bandwidth ( $\left.A v_{B W}\right)$}

In order to calculate bandwidth, the idle period of the wireless channel is a key parameter, which is determined by the traffic traveling along the mobile nodes as well as their neighborhoods. During that period the mobile nodes can successfully transmit data packets. The available bandwidth can be calculated as follows

$$
A v_{B W}=\operatorname{Max}{ }_{B W} *\left(\frac{I d l e_{t}}{I n t_{t}}\right)
$$

The carrier sense mechanism of IEEE 802.11 implemented in MANETs can judge whether the wireless channel is idle or busy and can be used to monitor the transition of channel state. In MAC layer, the virtual carrier sense mechanism can be used to determine whether channel is busy or idle. The period during which the channel changes its state from busy to idle in a unit time interval is known as Busy. Thus Idle $_{t}$ can be denoted by

Idle $_{\mathrm{t}}=\mathrm{Int}_{\mathrm{t}}-$ Busy $_{\mathrm{t}}$

Substituting the value of Idle $\mathrm{t}_{\mathrm{t}}$ into Equation (1), AvBW can be easily calculated [18].

\subsubsection{Computation of Residual Energy $\left(E_{R}\right)$}

After time $t$, the energy consumed by the node $E_{t}$ is calculated as follows. 
$\mathrm{E}_{\mathrm{t}}=\sigma * \mathrm{Tx}_{\mathrm{P}}+\rho * \mathrm{Rx}_{\mathrm{P}}$

Where $\operatorname{Tx}_{P}=$ Number of data packets transmitted by the node after time $\mathrm{t}$

$\mathrm{Rx}_{\mathrm{P}}=$ Number of data packets received by the node after time $\mathrm{t}$

$\sigma$ and $\rho$ are constants. Its value ranges between 0 and 1 .

If $E_{I}$ is the initial energy of a node, the residual energy $E_{R}$ of a node at time $t$, can be calculated as:

$\mathrm{E}_{\mathrm{R}}=\mathrm{E}_{\mathrm{I}}-\mathrm{E}_{\mathrm{t}}$

Substituting the value of Idle $\mathrm{t}_{\mathrm{t}}$ into Equation (1), AW can be easily calculated [18].

\subsubsection{Computation of Residual Energy $\left(E_{R}\right)$}

After time t, the energy consumed by the node $E_{t}$ is calculated as follows.

$\mathrm{E}_{\mathrm{t}}=\sigma * \mathrm{Tx}_{\mathrm{P}}+\rho * \mathrm{Rx}_{\mathrm{P}}$

Where $\operatorname{Tx}_{P}=$ Number of data packets transmitted by the node after time $\mathrm{t}$

$\mathrm{Rx}_{\mathrm{P}}=$ Number of data packets received by the node after time $\mathrm{t}$ $\sigma$ and $\rho$ are constants. Its value ranges between 0 and 1 .

If $E_{I}$ is the initial energy of a node, the residual energy $E_{R}$ of a node at time $t$, can be calculated as:

$E_{R}=E_{I}-E_{t}$

\subsubsection{Computation of Link Quality}

In most cases, the link quality can be characterized by signal strength, signal-to-noise ratio (SNR) or SINR. In our computation, we use SINR is used as the metric of link quality since it takes all the signal strength, interference and noise into account. Also, SINR directly influences bit error rate (BER) which determines the probability that a packet is successfully transferred. BER is inversely proportional to SINR, given a modulation method. SINR of the receiving signal is calculated by

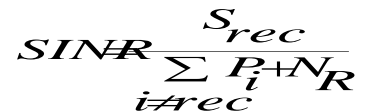

Where $S_{\text {rec }}$ is the received signal strength of the signal, $P_{i}$ denotes the individual received power of other signals received by the receiver simultaneously and $N_{R}$ is the effective noise at the receiver.

The Combined Weight $(\mathrm{CW})$ value based on the network parameters link quality, available bandwidth and residual energy is given by

$\mathrm{CW}=\mathrm{A} * \mathrm{Av}_{\mathrm{BW}+\mathrm{B}} * \mathrm{E}_{\mathrm{R}}+\mathrm{C} * \mathrm{SINR}$

Where A, B, C are normalization constants.

\subsection{QoS based Route Discovery}

\subsubsection{Route Request}

During the route discovery phase of the protocol, all the nodes in the network exchanges HELLO packets with each other. The source will add an extra CWM field with all its HELLO packets and broadcasts them to all other nodes. The CWM field contains the Source id (S), Destination id (D), Available Bandwidth $\left(\mathrm{Av}_{\mathrm{BW}}\right)$, Residual Energy $\left(\mathrm{E}_{\mathrm{R}}\right)$ and Link quality (SINR). Each node collects the bandwidth reserved at its one hop neighbors (piggybacked on periodic HELLO packets) and stores it in its Neighbor Table (NT).

To initiate QoS-aware routing discovery, the source host $\mathrm{S}$ sends a RREQ along with the Combined Weight Metric (CWM) value calculated using Eqn- 5 to the MA at neighboring nodes. When the Monitoring Agent (MA) receives the RREQ packet it compares its CWM value with the Minimum Combined Weight (MCW) value.

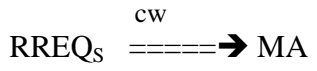

If $\mathrm{CW}$ value is higher than the $\mathrm{MCW}$, only then all the following copies of the Route Request are forwarded.

If another RREQ with higher CW is received, then the current $\mathrm{CW}$ is replaced by this higher value. This guarantees that the Route Request with the lowest congestion and maximum channel utilization is forwarded and employed for route creation.

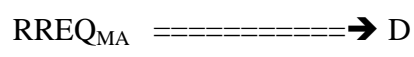

\subsubsection{Route Reply}

The Destination node D sends the route reply packet RREP to MA.

$$
\operatorname{RREP}_{\mathrm{D}}=============\rightarrow \mathrm{MA}
$$

Then MA forwards the route reply packet RREP to the S.

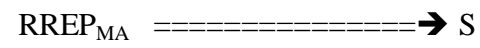

On receiving the RREP from the destination, the source selects the efficient route.

\section{ADAPTIVE RATE CONTROL WITH FAULT MANAGEMENT}

Our adaptive rate control protocol is dynamic weight adaptive with maximum and minimum values. Among a dynamic set of end-to-end flows in multihop wireless networks with dynamic channel conditions our adaptive rate control provides prioritized rate assurance and sophisticated bandwidth differentiation. The weight of each MAC flow adapts between a lower bound $\left(\mathrm{W}_{\mathrm{L}}\right)$ and an upper bound $\left(\mathrm{W}_{\mathrm{U}}\right)$. The $\mathrm{W}_{\mathrm{L}}$ is proportional to the rate requirement of the MAC flow, and the $\mathrm{W}_{\mathrm{U}}$ is proportional to the product of the rate requirement and the differentiating factor, which is larger for a MAC flow of higher priority, giving such a flow a higher $\mathrm{W}_{\mathrm{U}}$. The $\mathrm{W}_{\mathrm{L}}$ and the $\mathrm{W}_{\mathrm{U}}$ may change because of the rate requirement of a MAC flow which changes with the end-to-end flows. Each MAC flow periodically adapts its weight between the $\mathrm{W}_{\mathrm{L}}$ and the $\mathrm{W}_{\mathrm{U}}$ as prescribed in the algorithm-1. 
Let $F^{p}(i, j)$ be the MAC flow on link $(i, j)$ that carries the service class of priority $\mathrm{p}$. For the best-effort service class, $\mathrm{p}=$ 0 . For the QoS service classes, $\mathrm{p}=1,2,3 \ldots$ Let $\mathrm{W}^{\mathrm{p}}(\mathrm{i}, \mathrm{j})$ be the weight of flow $\mathrm{F}^{\mathrm{p}}(\mathrm{i}, \mathrm{j})$. Let $\mathrm{W}_{\mathrm{L}}^{\mathrm{p}}(\mathrm{i}, \mathrm{j})$ and $\mathrm{W}_{\mathrm{U}}^{\mathrm{p}}(\mathrm{i}, \mathrm{j})$ be the lower bound and upper bound for $\mathrm{W}^{\mathrm{p}}(i, j)$ respectively. Let $R_{R}^{p}(i, j)$ be the rate requirement of the MAC flow $F^{p}(i, j)$ and $R_{M}{ }^{p}(i, j)$ be the measured data rate of $F^{p}(i, j)$.

In order to compute $R_{R}{ }^{p}(i, j)$, we need to study two cases,

- In order to support the minimum rate requirement, we should allocate sufficient bandwidth for $F^{p}(i, j)$ provided an end-to-end flow carried by $\mathrm{F}^{\mathrm{p}}(\mathrm{i}, \mathrm{j})$ has a backlogged queue at $i$.

- The flow must have an upstream bottleneck, if the end-to-end flow does not have a backlogged queue and the arrival rate to the queue is smaller than its rate requirement. We only need to allocate enough bandwidth to cover the arrival rate.

The effective rate requirement of an end-to-end flow is equal to the minimum rate requirement in the first case and the arrival rate in the second case. We define $R_{R}{ }^{p}(i, j)$ as the summation of the effective rate requirements of all end-to-end flows carried by $F^{\mathrm{p}}(\mathrm{i}, \mathrm{j})$.

We define the $\mathrm{W}_{\mathrm{L}}$ and the $\mathrm{W}_{\mathrm{U}}$ for the weight $\mathrm{W}^{\mathrm{p}}(\mathrm{i}, \mathrm{j})$ of a MAC flow $F^{\mathrm{P}}(\mathrm{i}, \mathrm{j})$ as follows,

When $\mathrm{p}>0$,

$\mathrm{W}_{\mathrm{L}}^{\mathrm{p}}(\mathrm{i}, \mathrm{j})=\vartheta * \mathrm{R}_{\mathrm{R}}^{\mathrm{p}}(\mathrm{i}, \mathrm{j})$

$\mathrm{W}_{\mathrm{U}}^{\mathrm{p}}(\mathrm{i}, \mathrm{j})=\vartheta * \phi^{*} \mathrm{R}_{\mathrm{R}}^{\mathrm{p}}(\mathrm{i}, \mathrm{j})$

Where $\vartheta$ is a scaling coefficient, whose value can be set arbitrarily without changing the network's behavior and $\phi$ be the differentiating factor for priority $\mathrm{p}$.

When $\mathrm{p}=0$, the flow is best effort and we set the $\mathrm{W}_{\mathrm{L}}$ and the $\mathrm{W}_{\mathrm{U}}$ to be a fixed small value.

\section{Algorithm -1}

1. The monitoring agent (MA) at intermediate node $\left(\mathrm{N}_{\mathrm{i}}\right)$ of the MAC flow measures the incoming flow rate $\left(\mathrm{R}_{\mathrm{I}}\right)$ over its required rate $R_{R}$.

2. 1. If the $R_{I}<R_{R}$, then

2. 1.1. MA sends a positive acknowledgement (PACK) to the source

$$
\text { 2. 1. 2. If } R_{I}>R_{R} \text {, then }
$$

2.1. MA sends a negative acknowledgement (NACK) to the source

\section{If $\mathrm{S}$ receives PACK from MA then}

3. 1. S increases the weight of the flow at the end of each period until the $\mathrm{W}_{\mathrm{U}}$ is reached.

4. If $\mathrm{S}$ receives NACK from MA then
4. 1. S decreases the weight the flow at the end of each period until the $\mathrm{W}_{\mathrm{L}}$ is reached.

\subsection{Fault Management}

Link failure stems from node mobility and need of the network resources. Unlike fixed networks such as the Internet, QoS support in MANETs depends not only on the available resources in the network but also on the mobility rate of such resources. This is because mobility possibly will result in link failure which in turn may result in a broken path. Furthermore, MANETs potentially have fewer resources than fixed networks. Therefore, more criterions are required in order to capture the quality of the links between nodes.

Hence it is essential to capture the above mentioned characteristics to recognize the quality of links. Furthermore, the routing protocols must be adaptive to cope with the timevarying and low-capacity resources. For instance, due to the dynamic nature of the topology, it is possible that a route that was earlier found to meet certain QoS requirements no longer does so. In such a case, it is important that the network intelligently adapts the session to its new and changed conditions. Hence in order to avoid link failures, the monitoring agent (MA) at intermediate node $\left(\mathrm{N}_{\mathrm{i}}\right)$ measures CWM of each node $N_{i}$ at time $t_{k}$ and compare with the Minimum Combined Weight Metric (MCWM). If CWM value is lesser than MCWM, then a warning message which contains the id of $\mathrm{N}_{\mathrm{i}}$ is sent to the source, which then selects an alternate path.

\section{Algorithm -2}

1. The monitoring agent (MA) at intermediate node $\left(\mathrm{N}_{\mathrm{i}}\right)$ along the Destination D measures CWM of $\mathrm{N}_{\mathrm{i}}$ at time $\mathrm{t}_{\mathrm{k}}$.

\section{If the $\mathrm{CWM}<=\mathrm{MCWM}$, then}

2. 1. MA sends a warning message (WM) to the source which contains id of $\mathrm{N}_{\mathrm{i}}$

\section{If $\mathrm{S}$ receives $\mathrm{WM}$ from $\mathrm{MA}$ then}

3. 1. S discovers alternate path to the Destination (D).

\section{SIMULATION RESULTS}

\subsection{Simulation Setup}

The performance of Reliable and Robust QoS Routing Protocol (RRQRP) is evaluated through NS2 simulation [19]. A random network deployed in an area of $1000 \times 1000 \mathrm{~m}$ is considered. We vary the number of nodes as $50,75,100,125$ and 150. Initially the nodes are placed randomly in the specified area. The initial energy of all the nodes assumed as 10.1 joules. In the simulation, the channel capacity of mobile hosts is set to the same value: 2 Mbps. The distributed coordination function (DCF) of IEEE 802.11 is used for wireless LANs as the MAC layer protocol. The simulated traffic is CBR with UDP source and sink. The number of sources is varied from 1 to 4 . In our simulation, the speed is 5 $\mathrm{m} / \mathrm{s}$. and pause time is 5 seconds. 
Table 1 summarizes the simulation parameters used

\begin{tabular}{|l|l|}
\hline No. of Nodes & $50,75,100,125$ and 150 \\
\hline Area Size & 1000 X 1000 \\
\hline Mac & 802.11 \\
\hline Simulation Time & $50 \mathrm{sec}$ \\
\hline Traffic Source & $\mathrm{CBR}$ \\
\hline Packet Size & 512 \\
\hline Transmit Power & $0.660 \mathrm{w}$ \\
\hline Receiving Power & $0.395 \mathrm{w}$ \\
\hline Idle Power & $0.335 \mathrm{w}$ \\
\hline Initial Energy & $10.1 \mathrm{~J}$ \\
\hline Transmission Range & $250 \mathrm{~m}$ \\
\hline Rate & $\begin{array}{l}250,500,750 \text { and } 1000 \\
\mathrm{~kb}\end{array}$ \\
\hline
\end{tabular}

\subsection{Performance Metrics}

The performance of RRQRP is compared with QoS Based Routing protocol (QBRP) [9]. The performance is evaluated mainly, according to the following metrics.

5.2.1 Average end-to-end Delay: The end-to-end-delay is averaged over all surviving data packets from the sources to the destinations.

5.2.2 Average Packet Delivery Ratio: It is the ratio of the number of packets received successfully and the total number of packets transmitted.

5.2.3 Energy Consumption: It is the average energy consumption of all nodes in sending, receiving and forward operations.

5.2.4 Drop: It is the measure of number of packets dropped during a transmission.

The simulation results are presented in the next section.

\subsection{Simulation Results}

\subsubsection{Based on Nodes}

In our initial experiment, we vary the number of nodes as 50 , $75,100,125$ and 150 with traffic rate $250 \mathrm{~Kb}$.

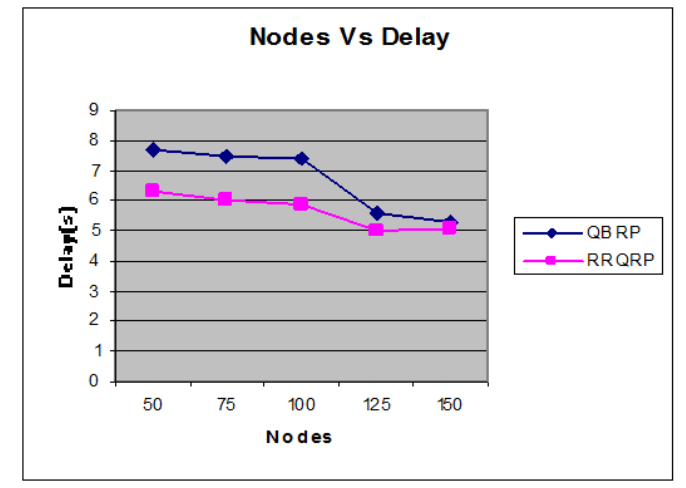

Fig-1: Nodes Vs Delay

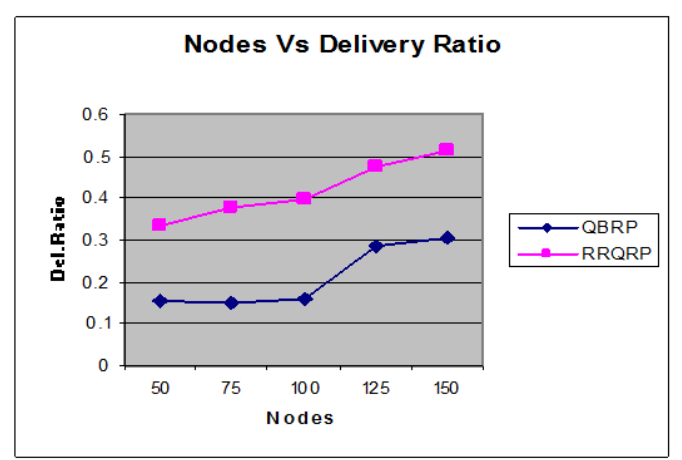

Fig-2: Nodes Vs Del ratio

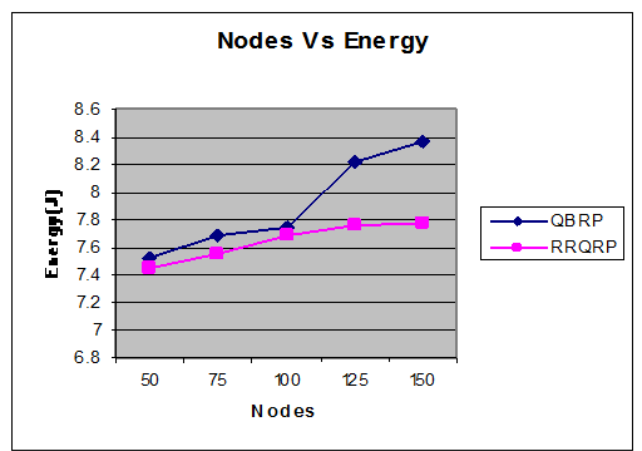

Fig-3: Nodes Vs Energy

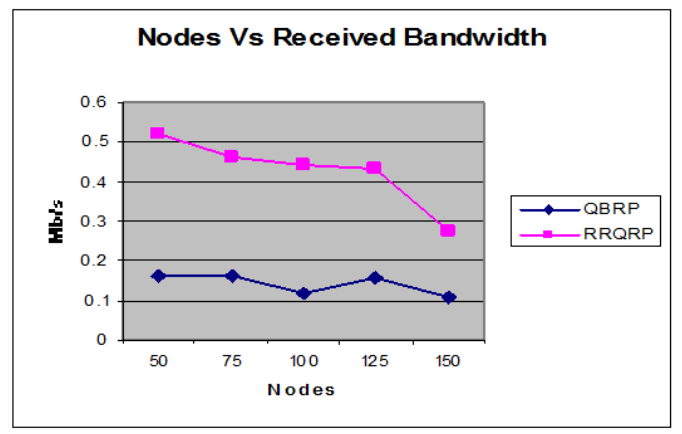

Fig-4: Nodes Vs Bandwidth

From Figure 1, we can see that the average end-to-end delay of the proposed RRQRP scheme is less when compared to the 
QBRP scheme. Figure 2 presents the packet delivery ratio of both the schemes. Since the packet drop is less and the throughput is more, RRQRP achieves good delivery ratio, compared to QBRP. Figure 3 shows the results of energy consumption. From the results, we can see that RRQRP scheme has less energy than QBRP scheme, since it has the energy efficient routing. Figure 4 shows the aggregated bandwidth of the schemes respectively. It is evident that in both the comparisons our proposed RRQRP is better than QBRP.

\subsubsection{Based on Rate}

In our second experiment, we vary the network traffic rates as $250,500,750$ and $1000 \mathrm{~kb}$ for fixed number of 50 nodes.

As the traffic rate is increased from $250 \mathrm{~kb}$ to $1000 \mathrm{~kb}$, the incoming traffic will also increase leading to the overflow of queue size. So the number of packet drops increases leading to the degradation of packet delivery ratio and increased endto-end delay. Since the rate is adjusted as dynamically as per the new sending rate at each sender, the performance of RRQRP outperforms QBRP in all the metrics.

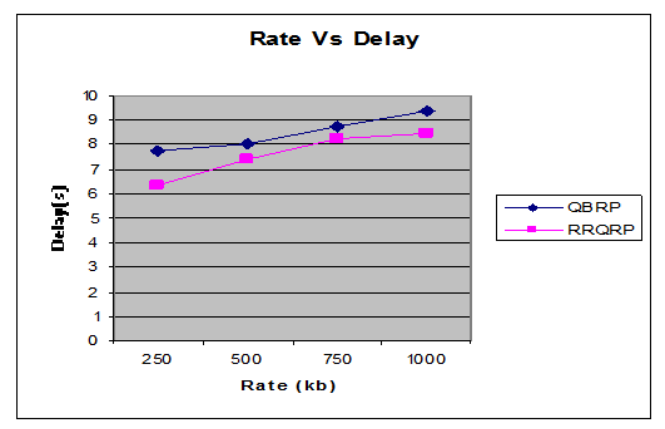

Fig-5: Rate Vs Delay

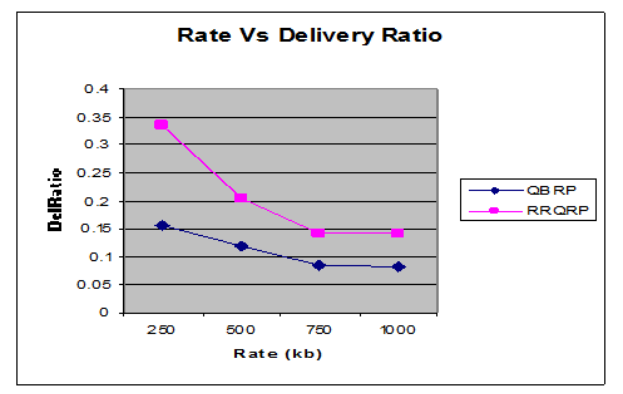

Fig-6: Rate Vs Packet Delivery ratio

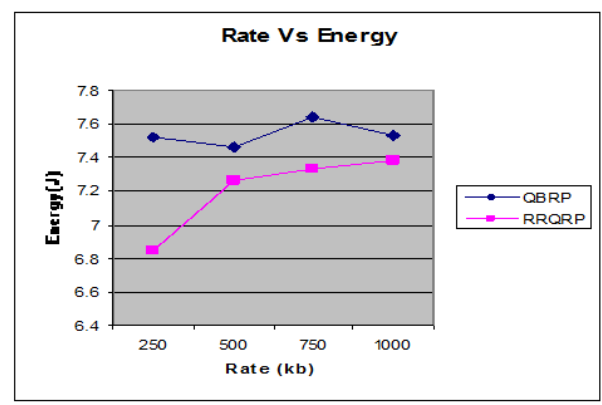

Fig-7: Rate Vs Energy

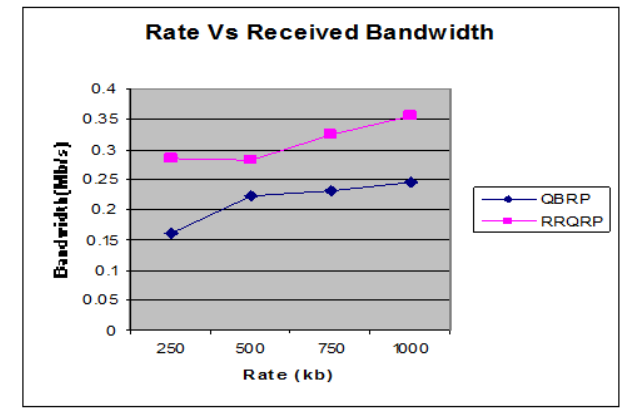

Fig-8: Rate Vs Received Bandwidth

From Figure 5, we can see that the average end-to-end delay of the proposed RRQRP scheme is less when compared to the QBRP scheme. Figure 6 presents the packet delivery ratio of both the schemes. Since the packet drop is less and the throughput is more, RRQRP achieves good delivery ratio, compared to QBRP. Figure 7 shows the results of energy consumption. From the results, we can see that RRQRP scheme has less energy than QBRP scheme, since it has the energy efficient routing. Figure 8 shows the aggregated bandwidth of both the schemes respectively. It is evident that in both the comparisons our proposed RRQRP is better than QBRP.

\section{CONCLUSION}

In this paper, we have proposed a reliable and robust QoS routing protocol for WSNs with mobile nodes, based on a Combined Weight $(\mathrm{CW})$ value. The $\mathrm{CW}$ is based on the network parameters such as link quality, residual energy and available bandwidth. Apart from this routing protocol, we follow an adaptive rate control mechanism in order to avoid congestion losses. Our adaptive rate control protocol is dynamic weight adaptive with lower bound and upper bound values, which provides prioritized rate assurance and sophisticated bandwidth differentiation among a dynamic set of end-to-end flows in multihop wireless sensor networks with dynamic channel conditions. Also in order to avoid link failures, the monitoring agent (MA) at intermediate node measures $\mathrm{CW}$ of each node and compare with the Minimum Combined Weight (MCW). If CW value is lesser than $\mathrm{MCW}$, then a warning message which contains the node id is sent to the source, which then selects an alternate path. By simulation results, we have shown that our proposed approach achieves high throughput with reduced energy consumption and delay, when compared existing routing protocol.

\section{REFERENCES}

[1] Bowman, M., Debray, S. K., and Peterson, L. L. 1993. Re1. John A. Stankovic," Wireless Sensor Networks" ieeexplore.ieee.org June 19, 2006.

[2] Yazeed Al-Obaisat, Robin Braun "On Wireless Sensor Networks: Architectures, Protocols, Applications, and Management" International Conference on Wireless ..., 2006.

[3] V. Bhoopathy, R. M. S. Parvathi, "Energy Efficient Secure Data Aggregation Protocol for Wireless Sensor Networks" European Journal of Scientific Research Vol.50 No.1 (2011), pp.48-58, 2011.

[4] Dazhi Chen and Pramod K. Varshney, "QoS Support in Wireless Sensor Networks: A Survey", International Conference on wireless sensor networks, 2004. 
[5] Mohamed A. el-Gendy, Abhijit Bose, Kang G.Shin, "Evolution of the Internet QoS and Support for Soft Real-Time Applications" IEEE conference, 2003.

[6] S.Muthukarpagam, V.Niveditta, S.Neduncheliyan, "Design issues, Topology issues, Quality of Service Support for Wireless Sensor Networks: Survey and Research Challenges", International Journal of Computer Applications (0975 - 8887) Volume 1 - No.

[7] Rajshree S.Dubey, Rajnish Choubey, Amit Dubey, "Challenges for Quality of Service (QoS) in Wireless Sensor Networks "International Journal of Engineering Science and Technology Vol. 2 (12), 7395-7400,2010.

[8] Kemal Akkaya and Mohamed Younis, "Energy and QoS aware Routing in Wireless Sensor Networks" Cluster Computing, 2005 - Springer.

[9] Mirela Fonoage, Mihaela Cardei, and Arny Ambrose, "A QoS Based Routing Protocol for Wireless Sensor Networks", 2010.

[10] Hakim Badis, Ignacy Gaw cedzki and Khaldoun Al Agha, "QoS Routing in Ad Hoc Networks Using QOLSR With no Need of Explicit Reservation" IEEE $60^{\text {th }}$ conference on Vehicular Technology Conference, 2004, Page(s): $2654-2658,2004$.

[11] M. K. Jeya Kumar, "Evaluation of Energy-Aware QoS Routing Protocol for Ad Hoc Wireless Sensor Networks", International Journal of Electrical and Electronics Engineering, vol. 4, 2010.

[12] Anelise Munaretto, Hakim Badi, Khaldoun Al Agha Guy Pujolle, "A Link-state QoS Routing Protocol for Ad
Hoc Networks" IEEE Conferences 4th International Workshop on Mobile and Wireless Communications Network, Page(s): 222 - 226 , 2002.

[13] Sanjay Kumar, Mayank Dave, Surender dahiya, "QoS based Solar Aware Clustered Routing for Wireless Sensor Networks" IJAST Vol No. 8, Issue No. 2, pp. 163 -168 .

[14] Deepali Virmani, Satbir Jain, "Stable Routing for achieving Quality of Service in wirelessSensor Networks" IJCA Special Issue on "Mobile Ad-hoc Networks" MANETs, 2010.

[15] Rui Dai, Pu Wang, and Ian F. Akyildiz, "CorrelationAware QoS Routing for Wireless Video Sensor Networks" Global Telecommunications Conference (GLOBECOM 2010), 2010 IEEE, pp. 1-5, 2010.

[16] Manuel Diaz, Eduardo Canete, Jaime Chen, "QoS aware and fault-tolerant routing for wireless Sensor and actor networks" 2011.

[17] Tenali. Nagamani, Damineni.SreeLakshmi," Improved Privacy in Wireless Sensor Network using QoS Routing Protocols" International journal of advanced engineering sciences and technologies Vol No. 9, Issue No. 1, 117 126, 2011.

[18] Fujian Qin and Youyuan Liu, "Multipath Routing for Mobile Ad Hoc Network", Proceedings of the 2009 International Symposium on Information Processing (ISIP'09), Huangshan, P. R. China, pp. 237-240, August 21-23, 2009.

[19] Network Simulator, http://www.isi.edu/nsnam/ns. 